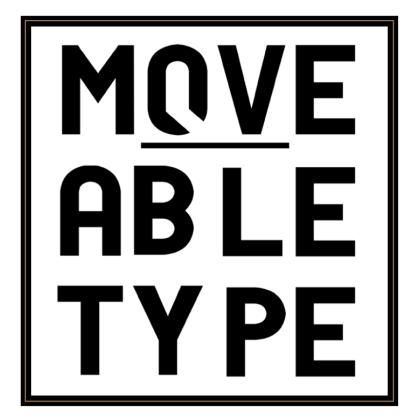

'Alien Investigations of Octopus Philosophies'

Author: K.V.K. Kvas

Source: Moveable Type, Vol.13, 'Ambience' (2021)

DOI: $10.14324 / 111.1755-4527.125$

MoveableType is a Graduate, Peer-Reviewed Journal based in the Department of English at UCL.

(C) 2021 K.V.K. Kvas. This is an Open Access article distributed under the terms of the Creative Commons Attribution License (CC-BY) 4.ohttps://creativecommons.org/licenses/by/4.0/, which permits unrestricted use, distribution, and reproduction in any medium, provided the original author and source are credited. 


\title{
Alien Investigations of Octopus Philosophies
}

\author{
K.V.K. Kvas
}

"As an octopus is dragged from its lair with a cluster of pebbles still clinging to the suckers, so Odysseus was swept from the rocks with the skin stripped from his desperate hands." - The Odyssey

The eight-armed oil-sleek-black head-body of the octopus spirals, twisting pseudo-octagonal traces of underwater splashless bubbleless waves, leaving in a blank blur blots of ink to the observer, in its stead. The inexact polygons within and without other polygons of pressure, fractals of turbulence, for the briefest moment, to the eye presuming itself to be anthropomorphic, may resemble the hexagons of a panelled library; or, of apiculturally perfected honeycomb. Water, like the sand of a beach whose glass or seaglass could not contain the hour of its passing through sifting fingers hungry for its fleeting silkiness, is a volatile graphic medium that leaves for neither word-painter nor the one who by reading rewrites, regraphs, no chance for fancies or pontifications about the purposeful endurance of bodily abjected collective memory or transgenerational territorial cultural pissings, whereas even the human art of paper marbling is designed for a shelf-life to outlive the water. The octopus, $a u$ contraire, is a philosopher of the moment whose philosophy corresponds exactly to, with, for that moment, only. The octopus leaves only, almost as byproduct, a vanishing-point, minimal trace, of its dynamic biochemical rhetoric whose cloudy shape-shifting toward eventual dissolution into the medium which other intelligences-despite having emerged countable generations ago from that very medium and so much more limited by self-involvement as to have expended centuries of similar bodily fluid leavings and exchanges battering materials mistaken for the transcendent ideas they were supposed to symbolize over the existence of such a thing as a "self" and their "selves" versus other often-deemed lesser "selves" - would mistake for a solid surface distinct from (for preserving) such injections. The octopus harbours no delusions about its significance beyond only partly-consciously calculated defense and camouflage, which repertoire can include phlegmatic pseudomorphic self-replicas signed 
as-it-were with a pseudonym, and autotomy (self-amputation) which the autonomous limbs of its decentralized nervous system survive to distract all but the most cannibalistic of impending predators (the Argonaut species detaches its penile arm for efficient deposition in the female's sometimes-gallery-of-a penis collection chamber; but nearly all species die anyway soon after mating). Even hyponomic jetting (where human anatomy conjoins the acts of breathing, eating-drinking (including ink-based seafood broth), and speaking, the octopus's mantle-syphon assemblage conjoins breathing, excreting, and locomotion, which also enables the dispersion of the melanin writings) at escape velocity risks pressuring a death-drive spiral of triple-heart-stopping hypoxia. Master of illusions (if it would deign to tolerate such illusions horrific as an honorific, or the tyranny of names in general, whose oppression lies precisely in that audacious disposal to attempt to solidify by the arbitrary formation of another material what is precisely anything but static, and which material may or may not even be more solid relative to the thing under-name), the octopus lets others deceive themselves otherwise by sensing it and narrating it, by telling tall tales of it to the vanishing point of mistaking them for its tales, tales told by it. And so "the" octopus, which cannot stand on its own eight feet save swimmingly, stands as tall as the dark shadow left pooling in its stead, in the heads steadfastly fascinated into fastening it to one of the many metaphors implanted there by the multifacetedness of its veil of camouflaged metamorphosis. When it clings to a rock or reef or seaweed, its skin may alter not only in its colour but in its very texture until, to an array of sensory capacities, only rock or reef or seaweed remains; until neither octopus nor rock seem to persist in what they are, but as phenomena whose stability is excluded from their conjunctive oscillation: extracting from octopus-or-rock only “or." Until blooming again through rainbows of reflections "back" to what it was, is, will be, will have been, would-should-could have been, and putting misconceptions about the blending powers of chameleonic distant relatives to shame. So as easily does it squeeze and stretch its rhetorical body through-with-as the circumstances. This cephalopod spaces out no time in philosophizing autolytically about the irresolvable, unreliable 
vagaries of sensorily limited appearances, skipping straight (though nonlinear or fusiform) instead to its unlimitedly fluid and complex disappearance through-by-as them, like the skins of negative space rippling beneath a tattoo-and through this, too: skeuomorphic ink impressed upon what once would have been the tanned and treated skins of animals, now the work of fossil oils hardened into button-capped keys for prehensile simian opposable digits' conversion into digits electrically blooming, at the touch, into pleasing (or malicious) disappearing acts of simulated inklings. For this being to "study" phenomenology (or teuthology) would be as redundant as the vehicle of camouflage itself relative to its metamorphic tenor.

Perhaps-perhaps (and the twofold fact that not only can we only say "perhaps" to introduce this idea but also that introducing such an idea in such a manner nonetheless paradoxically rings rhetorically an established sound of authority in that particular and peculiarly aposematic discourse of camouflage, probably taking its lineage from high-classed British stuffiness and the authority of that dialect whose imperialism is cited for its power to this day in Batesian human automated voice systems and advertisements)-perhaps anthropic distant relatives are not so different from the octopus, then, after all, except in one chief regard. Humans, too, are camouflage artists, as the ones they explicitly call "artists" perhaps best exemplify (but they are all artists and their privileging of only a select few with such a title is itself but an example of their art of camouflage and its chief difference and flaw), but the human art of camouflage may be so elaborate as to fool the artistes themselves (this seems its chief necessity)-so elaborate as to leave the human grasp altogether into something self-elaborating and auto-replicating quite apart from any human's delusion to control it, an organism of camouflage partly, and at least seemingly built by the human, yet parasitizing the human. The human builds and builds up its shell, its cave, its temple, its habitat, its environment, its imaginary, elaborated with every which mind-aping design of optical-illusion walls and ceiling outlining entire cosmologies of relative delusion; "entire" viewpoints of that elephant, the universe, become the object of the camouflage; from the smallest metaphors in the human's 
language to city-sized architectures and artistic displays that may or may not to some extent accurately macrocosmize them, the human inhabits its auto-deimatic illusion of materialized ideas with ideated material, and is consumed by them and/or consumes others in them, frequently those it mistakes as its own. While the human attempts to capture the image of the octopus and subsume it into this display or, at least, leave "immortalized" as its "legacy" the empire of dirt left dug from the Great Hole of Babel in which it unwittingly only secures its own burial beneath any epitaph-like signs of its passing (aiming for the dead cuttlefish's cuttle instead of the living cuttlefish's cutting phenomenowledge), the octopus indifferently (and probably usually unwillingly) contributes to the camouflageness-parasite that the human mistakes for its own; then squirts, leaves, hides, and waits to feed and/or fuck and/or eventually lay its pussy eggs. The camouflage parasite busily amuses its human with a mind's concerto of prayed imaginings of fast-feeding on a definition of the octopus; but the octopus slithers through and alongside as camouflage-itself on its way from prey to prey, entangling and suctioning. The human stops to stare in awe at its own ink leavings desecrating its unfurled red-carpet rug of prayer, tracing out in them a speculum of itself; but even tools, too (including memory and "for lack of a better word," the hubristic hypocrite will postpend - language) fall so within grasp of the Eightfold's intelligence. And what are tools, anyhow, to a creature already so well versed in the non-ecological false dichotomies of its organism's environmental "separation"?

The wink on my blink is ink, I think;

And the think-ink I think is cephalic.

In light (or rather dark) of all of the above - the Rorschach-like inkblots, macro-glyphs of the one they call Eightfold - I trust the Institute will now better understand the difficulty, futility, or impossibility of doing equal justice to the human, in turn, from the view of octopus phenomenology, as was tasked. All this is to say, such an endeavour ought to be viewed more in the ludic flavour of a childish indulgence. But perhaps that is speaking merely from the 
grandiose expectations set by Grand Natural Philosophy to begin with. If you would have some humility, you would have realized that every inch of the above was already dipped in the octopus's inkwell - and the only que ing is: How can I convince you that our alien phenomenology of the human from the ou of view produced a transcript rendering alien phenomenology of the octopus from the hum of view? How can I convince you of what I am

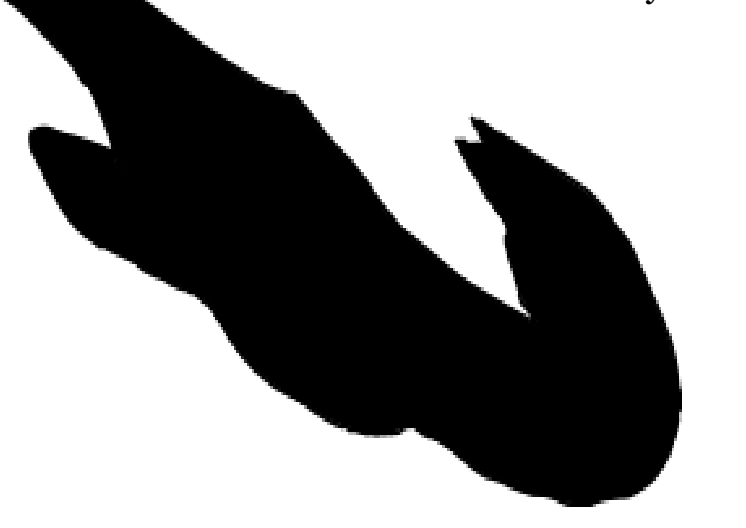

